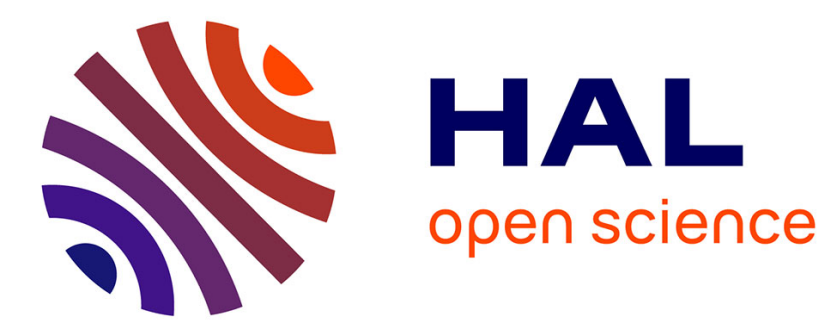

\title{
Les paradoxes de la querelle dans Antony and Cleopatra
}

\author{
Nathalie Vienne-Guerrin
}

\section{To cite this version:}

Nathalie Vienne-Guerrin. Les paradoxes de la querelle dans Antony and Cleopatra. Bulletin de la Société d'Etudes Anglo-Américaines des XVIIème et XVIIIème siècles, 2001, 53, pp.23-46. 10.3406/xvii.2001.1593 . halshs-01364015

\section{HAL Id: halshs-01364015 https://shs.hal.science/halshs-01364015}

Submitted on 11 Oct 2016

HAL is a multi-disciplinary open access archive for the deposit and dissemination of scientific research documents, whether they are published or not. The documents may come from teaching and research institutions in France or abroad, or from public or private research centers.
L'archive ouverte pluridisciplinaire HAL, est destinée au dépôt et à la diffusion de documents scientifiques de niveau recherche, publiés ou non, émanant des établissements d'enseignement et de recherche français ou étrangers, des laboratoires publics ou privés. 


\section{Les paradoxes de la querelle dans Antony and Cleopatra}

Nathalie Vienne-Guerrin

\section{Citer ce document / Cite this document :}

Vienne-Guerrin Nathalie. Les paradoxes de la querelle dans Antony and Cleopatra. In: XVII-XVIII. Bulletin de la société d'études anglo-américaines des XVIle et XVIIle siècles. N53, 2001. pp. 23-46;

doi : 10.3406/xvii.2001.1593

http://www.persee.fr/doc/xvii_0291-3798_2001_num_53_1_1593

Document généré le 24/05/2016 


\section{LES PARADOXES DE LA QUERELLE DANS ANTONY AND CLEOPATRA}

"Nay, but" (1.1.1): en plaçant ces deux termes en ouverture d'Antony and Cleopatra, ${ }^{1}$ Shakespeare met d'emblée la pièce sous le signe du débat ou de la discorde, l'un des fondements du tragique. ${ }^{2}$ Décrit ultérieurement comme "the word of war" $(2.2 .50 ; 3.1 .32)$, puis "the merèd question" (3.13.10), Antoine apparaît dès cette première scène comme au centre d'une controverse. La pièce entière peut être lue comme une gigantesque querelle - dans le sens juridique du terme - opposant deux parties, ${ }^{3}$ le dramaturge laissant la parole tantôt à l'accusation, tantôt à la défense des héros éponymes. Qu'elle apparaisse au gré des multiples commentaires émis par les personnages ou qu'elle soit véhiculée par la juxtaposition de scènes contrastées, cette opposition de points de vue donne à Antony and Cleopatra des allures de procès, faisant de cette cuvre une véritable "pièce à convictions," un tribunal où l'opinion est reine, mère de changements et de revirements spectaculaires, une cour de justice où la vérité échappe toujours. ${ }^{4}$ Les multiples frottements et différends qui animent la pièce semblent dès lors n'être que les reflets mouvants mais finalement invariables de cette macro-querelle. Signalée au fil du texte à de multiples reprises, ${ }^{5}$ la querelle s'accommode ici de tous les tons, de

1. Édition de référence: William Shakespeare, Antony and Cleopatra, ed. David Bevington (Cambridge: Cambridge UP, 1990).

2. Voir Yves Peyré, La voix des mythes dans la tragédie élisabéthaine (Paris: CNRS Éditions, 1996) 32-35.

3. Voir Thomas McAlindon, Shakespeare's Tragic Cosmos (Cambridge: Cambridge UP, 1991) 233.

4. "Antony and Cleopatra is a play haunted by the relativity of truth" (Peter J. Smith, Social Shakespeare. Aspects of Renaissance Dramaturgy and Contemporary Society [London: Macmillan, 1995] 85).

5. Voir les termes "wrangle" (1.1.50, 2.2.112), "quarrel" (1.3.66; 2.2.58), "square" (2.1.46; 3.13.41), "scrupulous faction" (1.3.48), "cleave" (3.4.31), "rift" (3.4.32), "scold" (1.1.34), "variance" (2.6.126), "conference harsh" (1.1.47), "chide" (1.1.51; 4.1.1), "taunt" (1.2.103; 2.2.77; 3.7.27), "dare" (1.2.177; 3.7.29-30; 3.13.25; 4.1.3), "division" (2.1.49), "difference" (2.1.50; 2.2.23), "private stomaching" (2.2.9), "stomach" (2.2.56; 3.4.12), "debate" (2.2.22), "curstness" (2.2.27), "contend" (2.2.86; 3.13.197), "offend"/"offence" 
toutes les formes, de toutes les échelles, de toutes les couleurs. L'expression "patch a quarrel"6 utilisée par Octave au cours de la scène qui réunit les triumvirs évoque l'image d'une querelle en forme de "patchwork" où des éléments variés sont cousus ensemble, image qui n'est pas sans nous renvoyer à la structure générale d'une pièce faite de "pièces," de morceaux assemblés dont l'unité repose sur la diversité. Au cœur des rapports amoureux et politiques, la querelle dresse des ponts et révèle des télescopages entre les domaines public et privé, laissant apparaître un réseau de motifs qui ne cessent de se faire écho d'un univers à l'autre.

Si l'on considère la structure de la pièce, il apparaît qu'elle repose sur une oscillation, un va-et-vient entre dispute et réconciliation. La querelle suit le parcours toujours identique et toujours changeant du flux et du reflux. Lorsque l'on connaît la prédominance de la contradiction dans cette pièce où le dramaturge n'hésite pas à accoupler, par exemple, les termes "boggler" et "ever" (3.13.113), créant ainsi le motif de la constante inconstance ou de l'éphémère permanence, il n'est pas surprenant de constater que l'art de la querelle y est un art paradoxal. Notre propos est d'étudier les principaux mécanismes de ce fonctionnement paradoxal. Nous verrons que, par sa rhétorique, la querelle épouse les battements contradictoires de la pièce, tant en elle se conjuguent l'excès et la modération. Mais la querelle apparaît également sous les traits de la "discordia concors" où l'éloge et l'injure se confondent et où les frontières entre guerre et compétition, discours érotique et discours guerrier tendent à disparaître. En ce sens la querelle est également emblématique d'une pièce où la dissolution et l'éclatement des contours se manifestent non seulement à l'occasion des méditations nébuleuses d'Antoine (4.14.1-22) mais aussi par le truchement des deux traits rhétoriques si souvent signalés: l'oxymore et l'hyperbole. La querelle semble alors à la fois omniprésente et absente dans cette pièce où l'expression "chercher querelle" peut se comprendre dans son sens propre. Elle peut être considérée comme un ressort dramatique mais également comme un moteur mis en marche de façon artificielle, avec des balbutiements, des ratés, des retards, mais qui fonctionne aussi sur le mode de l'éternel retour. À la fois toujours la même et toujours différente, la querelle se révèle être un lieu de réécriture privilégié.

(2.2.38; 2.5.101; 3.1.26; 3.2.33; 3.11.48; 4.15.47), "dispraise" (2.5.109), "wrestle" (3.2.63), "abused" (3.6.89), "traduced" (3.7.13), "rebuke" (3.7.25; 5.1.27), "challenge" (4.1.6), "rail" (1.2.103; 4.15.45), "strife" (2.2.86), "contend" (2.2.86).

6. "If you'll patch a quarrel, / As matter whole you have to make it with, / It must not be with this" (2.2.58-60). 


\section{Excès et nuance}

Dans Antony and Cleopatra, la querelle se décline sur le mode de l'excès, de l'inflation, mais aussi de la modération. Dès la première scène, l'esprit querelleur ("wrangling") est présenté comme l'un des visages, l'une des humeurs, l'un des "parfaits défauts" (2.2.241) de Cléopâtre:

Fie, wrangling queen,

Whom everything becomes, to chide, to laugh,

To weep, whose every passion fully strives

To make itself, in thee, fair and admired! (1.1.50-53)

Ce passage laisse déjà entrevoir en effet les perspectives paradoxales qui sont évoquées plus tard, notamment par Énobarbus, lorsqu'il décrit la rencontre des deux géants à Cydnus (2.2.200-55). Celle qui semble devoir défendre d'emblée son statut de reine d'Égypte ("As I am Egypt's queen" [1.1.31]) est décrite par Philon comme la reine des catins ("gipsy" [1.1.10], "strumpet" [1.1.13]), alors que les propos d'Antoine en font aussi une reine des mégères dont il faudrait brider la langue: "Let's not confound the time with conference harsh" (1.1.47). Cette dimension du personnage est confirmée lors de la confrontation entre Antoine et Cléopâtre, quand celle-ci apprend que son général doit partir "pour cause de décès." Antoine se révèle alors incapable de contenir cette langue rebelle. "Quarrel no more" (1.3.66), "You'll heat my blood. No more" (1.3.80), "Now by my sword" (1.3.82): ces injonctions restent sans effet jusqu'à ce qu'Antoine menace finalement de partir. Cette figure de mégère qui apparaît dès la première scène est prolongée par les figures de la putain ${ }^{7}$ et de la sorcière ${ }^{8}$ si souvent évoquées au cours de la pièce, comme pour refléter l'amalgame que les Élisabéthains faisaient entre ces profils féminins. ${ }^{9}$ Dans l'imaginaire de la Renaissance, il n'y a qu'un pas à faire entre les deux créatures béantes que sont la catin et la mégère, tandis que la violence verbale est le principal attribut qụe la mégère et lạ sorcière ont en commun. Le dramaturge entretient tout au long de la pièce l'image d'une Cléopâtre que l'on tente de dompter... en vain. Incarnation de la querelle et source de désordre, elle semble avoir les attributs de la

7. "Gipsy" (1.1.10; 4.12.28), "strumpet" (1.1.13), "salt Cleopatra" (2.1.21), "your fine Egyptian cookery" (2.6.63), "his Egyptian dish" (2.6.123), "whore" (3.6.69), "trull" (3.6.99), "yon ribaudred nag of Egypt" (3.10.10), "kite" (3.13.91), "boggler" (3.13.113), "a morsel cold upon / Dead Caesar's trencher" (3.13.119-20), "a fragment / Of Cneius Pompey" (3.13.120-21), "triple-turned whore" (4.12.13), "whore" (5.2.220).

8. "this enchanting queen" (1.2.125), "this great fairy" (4.8.12), "my charm" (4.12.16), "thou spell" (4.12.30).

9. Il n'est qu'à voir le titre de l'ouvrage de Joseph Swetnam, The Araignment of Lewde, Idle, Froward, and Unconstant Women (London, 1615), STC 23533. Ce titre résonne comme une description de la Cléopâtre shakespearienne. 
mégère. C'est une "mauvaise langue" qui n'a sans doute rien à envier à Fulvie qu'elle décrit comme une harpie criarde ("When shrill-tongued Fulvia scolds" [1.1.34]), ${ }^{10}$ semblant ainsi dresser un autoportrait dont on goûtera toute l'ironie. La reine est rarement le "rossignol" ("Nightingale" [4.8.18]) évoqué par le général déchu mais apparaît en revanche régulièrement au cours de la pièce comme une voix discordante, médisante, maudissante, grinçante. La figure du serpent à laquelle elle est associée ("my serpent of old Nile" [1.5.26]) peut contribuer à mettre en relief cette facette du personnage. ${ }^{11}$ Les propos désobligeants qu'elle tient à l'encontre de ses rivales Fulvie $^{12}$ et Octavie $^{13}$ font d'elle une Mégère dans le sens mythologique du terme, c'est-à-dire une "Jalouse." ${ }^{14}$ Les sarcasmes obscènes qu'elle décoche à l'eunuque Mardian ${ }^{15}$ la confortent dans son statut de maîtresse-femme, ou pour reprendre l'expression de Natalie Zemon Davis de "Woman on Top." 16 La scène au cours de laquelle elle apprend le mariage d'Antoine et d'Octavie la fait apparaître comme une véritable Furie. Elle devient cette "gorge mal disante"17 ("illuttering throat" [2.5.35]) dont les malédictions semblent pouvoir conférer au Verbe un pouvoir magique. Cléopâtre incarne alors les excès de la langue. "I do not like 'But yet'" (2.5.51), "Fie upon 'But yet'!" (2.5.52): ces quelques mots peuvent résumer à eux seuls la philosophie du langage représentée ici par Cléopâtre, philosophie que tout semble contredire dans cette pièce qui n'est que "but yet," c'est-à-dire qui cultive le point de vue et l'ambiguiité. Le refus de la nuance exprimé par Cléopâtre a pour prolongement l'amour de l'hyperbole, cette passion de l'excès qui trouve peut-être l'une de ses plus criantes manifestations dans le flot de malédictions déversé notamment au cours de cette scène où, dans sa violence, Cléopâtre joint le geste à la parole:

The most infectious pestilence upon thee!

Strikes him down (2.5.62)

10. Cléopâtre fait des commentaires sur la voix de Fulvie (1.1.34), d'Octavie (3.3.1216) mais aussi d'Antoine (5.2.82-85).

11. Sur la symbolique du serpent, voir l'article de Ladan Niayesh, "Les Serpents d'Antoine et Cléopâtre," Lectures de Shakespeare. Antony and Cleopatra, ed. JeanChristophe Mayer (Rennes: PU Rennes, 2000) 115-23.

12. Voir 1.1.22, 30, 34; 1.3.20-23.

13. Voir toute la scène 3 de l'acte 3.

14. Voir Dictionnaire Historique de la Langue Française (Paris: Le Robert, 1992) article "Mégère."

15. 1.5.10-13 et 2.5.5-9.

16. Natalie Zemon Davis, Society and Culture in Early Modern France, 1965 (Cambridge: Polity P, 1998) 124-51 (chapitre 5).

17. Traduction de Henri Thomas, Antoine et Cléopâtre, 1959 (Paris: Flammarion, 1996). 
Hence,

Horrible villain, or I'll spurn thine eyes

Like balls before me! I'll unhair thy head!

She hales him up and down

Thou shalt be whipped with wire, and stewed in brine,

Smarting in lingering pickle! (2.5.63-67)

Melt Egypt into Nile, and kindly creatures

Turn all to serpents! (2.5.79-80)

The merchandise which thou hast brought from Rome

Are all too dear for me. Lie they upon thy hand

And be undone by 'em! (2.5.104-106)

Ces imprécations qui se développent en forme de chapelets deviennent une litanie sans fin. En matière de malédictions comme de prières, c'est la répétition qui est de mise. Toute la scène repose sur cette structure répétitive. Confondant le messager et son message, Cléopâtre entreprend un dépeçage symbolique tandis que le corps devient l'ingrédient d'une cuisine infernale digne d'un Jérôme Bosch. L'information transmise par le messager devient un "casus belli" et celuici fait alors office de tampon. Le héraut se substitue au héros. Lorsque Cléopâtre, lors d'une courte accalmie, lui demande si son histoire "tient" toujours ou s'il "s'en tient" à cette version ("The gods confound thee, dost thou hold there still?" [2.5.94]), elle manifeste une croyance dans le pouvoir magique du mot. On entrevoit alors, mais pour un instant seulement, l'idée que les mots puissent défaire les actes. César met en relief l'épaisseur sonore du personnage lorsqu'il décrit Cléopâtre comme "a trull / That noises it against us" (3.6.99-100) et à la mort d'Antoine, la reine se dit prête à tempêter si fort ("rail so high") que la Fortune en sortira de ses gonds (4.15.45-47). Ce que ces éclats de voix révèlent de Cléopâtre, Shakespeare s'emploie à le développer sur le mode symbolique. Les renversements ou tout au moins les brouillages génériques qui sont au cœur de la pièce traduisent la monstruosité attachée au statut de la mégère qui met sens dessus dessous les rapports entre le féminin et le masculin. ${ }^{18}$ Si Cléopâtre s'exclame avec extase "O happy horse, to bear the weight of Antony!" (1.5.22), évoquant ainsi un rapport "naturel" entre les deux sexes, la pièce laisse pourtant entrevoir à

18. Voir notamment Janet Adelman, Suffocating Mothers (London: Routledge, 1992) 174-92; Jean-Pierre Maquerlot, "Masculin-féminin dans Antoine et Cléopâtre," Lectures d'une auvre: Antony and Cleopatra, ed. Christine Sukic (Paris: Éditions du Temps, 2000) 59-75. 
de nombreuses reprises ${ }^{19}$ l'image d'une femme dominatrice qui pourrait bien ressembler à cette harpie que les illustrations de l'époque représentent à califourchon sur le dos de son époux. ${ }^{20}$ Le sort que César mais aussi Antoine (4.12.32-39) semblent réserver à la reine d'Égypte s'apparente par ailleurs aux humiliations publiques que la société élisabéthaine infligeait aux mégères en les trimballant à travers la ville en charrette sous les huées des autres habitants. Le terme "a-ducking" (3.7.64), ${ }^{21}$ qu'un soldat romain associe aux Phéniciens mais aussi aux Égyptiens et donc à leur reine, évoque la cruelle tradition populaire qui consistait à imposer à ces fauteuses de trouble un bain forcé après les avoir installées sur un trône souvent percé ("ducking-stool" ou "cuckingstool"). ${ }^{22}$

Mais si l'on peut réduire Fulvie et Octavie à un seul ton, une seule voix ("shrill-tongued" [1.1.34] ou "low-voiced" [3.3.13]), Cléopâtre quant à elle ne peut être inscrite dans le seul registre de la mégère. ${ }^{23}$ Le portrait débordant quoique lacunaire qu'Énobarbus dresse d'elle trouve son contrepoint lors de l'interrogatoire de l'acte 3 , scène 3 , où les êtres semblent pouvoir appartenir à une seule catégorie de taille ("dwarfish"

19. Voir notamment la fusion dramatique des deux personnages lorsque Énobarbus croit voir Antoine alors qu'il s'agit de Cléopâtre (1.2.71-72). Voir aussi le commentaire de César (1.4.5-7), l'échange des habits évoqué par Cléopâtre (2.5.22-23), les propos d'Énobarbus (3.7.10-15) et de Canidius (3.7.70) qui se plaignent d'être sous la coupe d'une femme. Voir aussi l'image d'Antoine accroché à sa reine lors de la bataille d'Actium (3.11.55-67).

20. Voir par exemple lillustration qui apparaît en frontispice de The Brideling, Sadling and Ryding, of a rich Churle in Hampshire (London, 1595), STC 19855.

21. Seule occurrence du terme dans tout le corpus shakespearien. Qu'Antoine veuille aller "barboter," voilà qui fait partie du processus de "féminisation" du personnage que de nombreux critiques ont décelé dans cette pièce.

22. À propos du sort réservé aux mégères, voir David E. Underdown, "The Taming of the Scold: the Enforcement of Patriarchal Authority in Early Modern England," Order and Disorder in Early Modern England, ed. Anthony Fletcher et John Stevenson (Cambridge: Cambridge UP, 1985) 116-39. Dans "'Scolding women cucked or washed': a crisis in gender relations in early modern England?," Women, Crime and the Courts, ed. John Frank Kermode et Garthine Walker (London: UCL P, 1994) 48-80, Martin Ingram remet en cause la plupart des affirmations de Underdown. Voir aussi l'article de Lynda $E$. Boose, "Scolding Brides and Bridling Scolds: Taming the Woman's Unruly Member," Shakespeare Quarterly 42 (1991): 179-213. Voir également Vienne-Guerrin, "La réécriture de la Mégère dans le théâtre de Shakespeare," Réécritures, ed. Maquerlot (Rouen: PUR, 2000) 77-92.

23. Pour cette variété de tons, voir Geoffrey Bullough, Narrative and Dramatic Sources of Shakespeare (London: Routledge, 1964) 273-75. Plutarque parle de "the excellent grace and sweetness of her tongue," mais évoque aussi les railleries de Cléopâtre avant de la décrire comme suit: "her tongue was an instrument of musicke to divers sports and pastimes, the which she easily turned to any language that pleased her." 
[3.3.16]), de voix ("low-voiced" [3.3.13]), de forme ("long or rond?" [3.3.29]) ou de couleur ("brown" [3.3.33]). Le catalogue autour duquel s'organise le portrait-robot d'Octavie rompt avec la description finalement vide d'Énobarbus qui révèle le caractère inclassable de la reine d'Égypte. ${ }^{24}$ On sent ici le gouffre existant entre la caricature et le portrait. Contrairement à Fulvie ou Octavie, Cléopâtre ne peut s'en tenir à une seule voix. La langue tapageuse que nous venons de décrire a pour contrepoint des silences très frappants, notamment lorsque c'est au tour d'Antoine de laisser libre cours à ses imprécations. Quand la voix de l'un faiblit, c'est celle de l'autre qui se fait entendre. Les silences de l'un sont habités par les cris de l'autre, le jeu des voix suivant lui aussi le schéma du flux et du reflux en une succession de piano et de forte. Antoine apparaît en effet lui aussi à plusieurs reprises comme un être qui gronde, une voix tonitruante ("as rattling thunder" [5.2.85]). Shakespeare souligne de manière récurrente ce que Plutarque évoquait en parlant de "his outrageous manner of railing. " 25 Avant la confrontation entre Antoine et Octave, Énobarbus espère que son général parlera "aussi haut que Mars" ("speak as loud as Mars" [2.2.6]); lors de la scène où il croit surprendre Cléopâtre en train d'accorder ses faveurs à Thidias, Antoine aimerait mugir "plus fort que le troupeau cornu"26 ("outroar / The hornèd herd" [3.13.130-31]) comme si ses exclamations et ses malédictions étaient vouées à être toujours insuffisantes. Rejoignant le mythe de "Hercules Furens," Antoine apaise ses souffrances et ses peurs ${ }^{27}$ dans l'excès et vide ses querelles politico-domestiques comme on vide un abcès, en laissant couler tout le fiel qu'il contient. La querelle s'épuise dans la destruction de l'autre et dans l'autodestruction. Les injures qu'il lance à la face de Cléopâtre font partie d'une entreprise systématique de démolition. La reine n'a plus de nom: "she here - what's her name / Since she was Cleopatra" (3.13.100-101). Les mots d'Antoine la flétrissent ("you were half blasted ere I knew you" [3.13.107]), la dépècent puisqu'elle n'est plus qu'un morceau ("morsel" [3.13.119]), qu'un fragment ("fragment" [3.13.120]). Ce dépeçage symbolique trouve son pendant concret dans la mutilation infligée à Thidias au cours d'un épisode qui n'est que le miroir de l'acte 2 , scène 5 . On a ici affaire à une scène de flagellation verbale autant que physique. Cependant Antoine n'est satisfait ("satisfied"

24. Voir François Laroque, "Antoine et Cléopâtre ou l'esthétique du vide," Études Anglaises 53.4 (2000): 400-12.

25. Cité dans Bullough 272-73.

26. Traduction de Thomas.

27. Voir la remarque d'Énobarbus "To be furious / Is to be frighted out of fear" (3.13.199-200). 
[3.13.171]) que lorsque Cléopâtre le rejoint dans cette destruction symbolique en se maudissant elle-même sur un mode hyperbolique bien qu'hypothétique:

Ah, dear, if I be so,

From my cold heart let heaven engender hail,

And poison it in the source, and the first stone

Drop in my neck; as it determines, so

Dissolve my life! The next Caesarion smite,

Till by degrees the memory of my womb,

Together with my brave Egyptians all,

By the discandying of this pelleted storm

Lie graveless till the flies and gnats of Nile

Have buried them for prey! (3.13.162-71)

Elle mobilise alors tout un imaginaire apocalyptique, comme s'il fallait faire table rase d'un monde pour pouvoir en reconstruire un autre. La mort symbolique de Cléopâtre et de l'univers qu'elle représente préfigure la fausse annonce de sa mort qui apaisera plus tard la colère d'Antoine. Dans une pièce où les personnages sont "bons" une fois qu'ils ne "sont plus" ("She's good, being gone" [1.2.123]), il n'est pas étonnant que la querelle ne puisse s'épuiser que dans la mort. Enfin, après la prétendue trahison de Cléopâtre, Antoine laisse une fois de plus libre cours à ses excès de langue et déverse sur l'infâme Égyptienne ("this foul Egyptian" [4. 12.10]) un flot d'injures et de malédictions: "Triple-turned whore" (4.12.13), "this false soul of Egypt" (4.12.25), "thou spell" (4.12.30), "the witch" (4.12.47), où le général épouse la vision romaine de Cléopâtre qui apparaissait dès la première scène. La malédiction qu'il lui lance (4.12.33-39) préfigure d'ailleurs le sort humiliant auquel la Reine voudra échapper à la fin de la pièce. ${ }^{28}$ Lorsque, dans un moment de calme après la tempête, Cléopâtre se réjouit de retrouver son Antoine ("but since my lord / Is Antony again" [3.13.190-91]), on entrevoit l'idée que l'on se perd soi-même dans l'excès de langage. De même, lorsque Charmian enjoint à sa maîtresse de se calmer par un "keep yourself within yourself" (2.5.76), ses propos semblent suggérer que Cléopâtre n'est plus elle-même ou qu'elle est "hors d'elle" lorsqu'elle se laisse aller à l'invective. Éclat de voix et éclat de soi vont de pair et il semble que l'on se dissout dans l'explosion verbale. Cependant il est quelque peu ironique de demander à Cléopâtre, personnage protéiforme, de ne pas être "hors d'elle" ou de se féliciter d'avoir retrouvé le "vrai" Antoine, dans une pièce

28. Cette exécration hante Cléopâtre jusqu'à la fin de la pièce puisqu'elle mobilise à deux reprises les mots d'Antoine (5.2.54-56 et 5.2.206-220). Le suicide de Cléopâtre montre qu'elle donne valeur de prédiction à cette malédiction. 
où les deux protagonistes sont toujours "hors d'eux," soit parce que, comme Cléopâtre, ils échappent à toute description, soit parce que, comme Antoine, ils ne sont plus ce qu'ils ont été ou ne savent plus ce qu'ils sont.

Les querelles ouvertes que nous venons d'analyser ne sont cependant que les versions hyperboliques de la querelle plus feutrée qui couve entre Antoine et Octave. Les propos échangés par Énobarbus et Lépide en préambule de la confrontation des triumvirs laissent apparaître deux conceptions de la querelle:

LEPIDUS

Good Enobarbus, 'tis a worthy deed,

And shall become you well to entreat your captain

To soft and gentle speech.

ENOBARBUS

I shall entreat him

To answer like himself. If Caesar move him,

Let Antony look over Caesar's head

And speak as loud as Mars. By Jupiter,

Were I the wearer of Antonio's beard,

I would not shave't today.

LEPIDUS

'Tis not a time for private stomaching. (2.2.1-9)

En opposant les points de vue de Lépide et d'Énobarbus, Shakespeare oppose raison et passion ("your speech is passion" [2.2.13]), excès et modération, querelle privée et intérêt public. Dans l'espace diplomatique, la querelle doit être réglementée. Lépide se présente au cours de cette scène comme le contrôleur des langues qui transmet tout d'abord des consignes de bonne conduite verbale:

LEPIDUS

What's amiss,

May it be gently heard. When we debate

Our trivial difference aloud, we do commit

Murder in healing wounds. Then, noble partners,

The rather for I earnestly beseech,

Touch you the sourest points with sweetest terms,

Nor curstness grow to th' matter. (2.2.21-27)

En véritable arbitre, ${ }^{29}$ il s'emploie à brider les propos de César ("Soft, Caesar!" [2.2.90]), avant de rendre son verdict: "'Tis noble spoken"

29. Lépide est également décrit comme un pacificateur par un serviteur: "As they pinch one another by the disposition, he cries out, No more', reconciles them to his entreaty, and himself to the drink" (2.7.5-7). 
(2.2.105), "Worthily spoken, Maecenas" (2.2.109). La querelle est ici encadrée, canalisée par un agent modérateur et devient une procédure judiciaire. À chaque accusation répond une défense et les motifs de dispute sont ainsi successivement évacués. "I learn you take things ill which are not so" (2.2.35), "how intend you, 'practis'd'?" (2.2.46), "You do mistake your business" (2.2.51): la querelle est ici affaire d'interprétation. La récurrence du mot "if ${ }^{30}$ la plonge dans le domaine de l'hypothétique. Le terme fonctionne comme un amortisseur, "a peacemaker," pour reprendre le terme de Touchstone dans Comme Il Vous Plaira ("Your 'if' is the only peacemaker" [5.4.97-98]): ${ }^{31}$ "Yet if you there / Did practise on my state, your being in Egypt / Might be my question" (2.2.44-46).

On sent derrière cette scène la présence des codes du duel qui permettent d'échapper à l'affrontement ${ }^{32}$ ou qui garantissent une querelle "juste" ("A Fair Quarrel"). ${ }^{33}$ Là où Antoine et Cléopâtre cultivent l'excès, les triumvirs cultivent la nuance: Antoine tient à substituer au "denied" de César un "neglected" (2.2.96) plus consensuel. Les parties en présence cultivent le "mais pourtant," le "but yet" (2.5.51-52) que Cléopâtre rejette quelques scènes plus tard. Les mêmes procédés d'agression indirecte interviennent lors de l'échange acerbe entre les triumvirs et Pompée. Aux excès que nous avons décrits plus haut, les chefs de guerre préfèrent les sous-entendus et les non-dits, aux "gros mots," ils préfêrent les demimots. C'est à demi-mot que Pompée reproche à Antoine d'avoir volé le nid de son père en évoquant l'image du coucou (2.6.28); c'est à demi-mot qu'il évoque le passé sulfureux de Cléopâtre (2.6.63-65) titillant ainsi la corde sensible du général romain. En appelant les triumvirs "the senators alone of this great world" (2.6.9), il habille la critique de termes apparemment flatteurs. ${ }^{34}$

Dans Antony and Cleopatra, Shakespeare met donc en scène deux pratiques de la querelle: l'une débridée, l'autre réglementée, l'une sauvage, l'autre policée, l'une passionnée, l'autre diplomatique, l'une spectaculaire, l'autre discrète, l'une égyptienne, l'autre romaine. Cependant, une querelle peut en cacher une autre. Toutes deux sont

30. 2.2.4, 16, 37, 44, 58, 86, 106, 110, 121, 129, 151.

31. Shakespeare, As You Like It, ed. Alan Brissenden (Oxford: Oxford UP, 1993).

32. À ce sujet, voir Vienne-Guerrin, "La réécriture des codes de duel dans l'injure shakespearienne," Réécritures, ed. Maquerlot (Rouen: PU Rouen, 2000) 37-53.

33. Voir la pièce de Thomas Middleton et William Rowley, A Fair Quarrel (1617), ed. Roger V. Holdsworth (London: Ernest Benn, 1974).

34. Voir note de David Bevington, 137: "i.e. sole leaders of the state; but the choice of words implies a criticism that the triumvirs have supplanted the senators of Rome, whose republican traditions Pompey purports to represent." 
l'expression d'un monde bâti sur une harmonieuse discorde. Shakespeare s'emploie non seulement à dessiner mais aussi à gommer ces frontières et fait de ces querelles "un divin mélange" ("heavenly mingle" [1.5.62]) en brouillant notamment les limites entre le domaine privé et le domaine public, entre la concorde et la discorde, entre l'aigre et le doux. "Touch you the sourest points with sweetest terms" (2.2.26): ce décret émis par Lépide peut être renversé en une formule qui définirait tout aussi bien ce qu'est la querelle dans cette pièce: "Touch you the sweetest points with sourest words."

\section{La discorde harmonieuse}

En mettant en scène le mariage mythique de Vénus et de Mars, ${ }^{35}$ Shakespeare place au cœur de sa pièce le motif de la "discordia concors," de la réconciliation des contraires. Notre propos est de montrer comment la querelle, telle qu'elle est pratiquée dans cette pièce, est une manifestation exemplaire de cette discorde harmonieuse. Elle fait tout d'abord partie du jeu érotique, comme le suggère l'échange entre Charmian et Cléopâtre:

\section{CHARMIAN \\ Madam, methinks if you did love him dearly; \\ You do not hold the method to enforce \\ The like from him. \\ Cleopatra \\ CHARMIAN \\ What should I do I do not? \\ In each thing give him way. Cross him in nothing. \\ Cleopatra \\ Thou teachest like a fool: the way to lose him. \\ CHARMIAN \\ Tempt him not so too far. I wish, forbear; \\ In time we hate that which we often fear. (1.3.6-12)}

Ce passage constitue une théorisation des mécanismes du désir. Si l'amour selon Charmian naît de la concorde, l'amour selon Cléopâtre vit de contrariétés. On a ici affaire à une véritable querelle d'idées. Mais si la position de Charmian semble aller à l'encontre de celle de sa maîtresse, son discours est cependant travaillé par le contre-discours de Cléopâtre puisque le terme "tempt" unit désir (tentation) et contrariété ("tempt"

35. Voir Adelman, The Common Liar (New Haven: Yale UP, 1973) 91-92; McAlindon, Shakespeare and Decorum (London: Macmillan, 1973) 195; McAlindon, Cosmos 230-34; Peyré 32-49, 233-35, 261-62. 
signifiant "try," vex"). Cette querelle sur les bienfaits et les limites de la querelle révèle une concorde derrière la discorde. L'amphibologie du terme "tempt" permet à des discours opposés de se rejoindre. Le mélange d'accord et de désaccord apparaît donc à la fois dans le contenu et dans la forme de cet échange. Mais la mise en garde de Charmian ("Tempt him not so too far") constitue également une réflexion sur la gestion de la limite. En matière de querelle, il est des bornes à ne pas dépasser sous peine de la voir dégénérer en guerre ouverte ou en divorce. La querelle est une affaire d'équilibre et le dramaturge s'emploie tout au long de la pièce à mettre cet équilibre en danger en jouant constamment sur les limites dans le cadre des rapports entre Antoine et Cléopâtre mais aussi entre Antoine et César. Cet équilibre de la querelle trouve sa manifestation rhétorique dans le premier échange entre les deux héros éponymes:

\section{Cleopatra}

If it be love indeed, tell me how much. ANTONY

There's beggary in the love that can be reckoned. Cleopatra

I'll set a bourn how far to be beloved. ANTONY

Then must thou needs find out new heaven, new earth. (1.1.14-17)

Cette querelle initiale fondée sur une opposition de points de vue constitue le parfait exemple de ce que nous entendons par "discorde harmonieuse." L'usage de la stichomythie qui structure le dialogue permet au dramaturge de créer un équilibre entre accord et désaccord. Le parallélisme des vers met en effet en relief le mariage mais aussi le conflit des deux parties en présence, l'une incarnant la mesure, l'autre la démesure. Un peu plus loin, l'utilisation du vers partagé ("Antony / Will be himself / But stirred by Cleopatra" [1.1.44-45]) produit le même effet en exprimant un mélange de collaboration et d'opposition. Celle qui prétend pouvoir définir les limites ("I'll set a bourn how far to be beloved") sait pousser la querelle jusqu'à son comble, rendant ainsi extatiques l'apaisement et la satisfaction ("satisfied" [3.13.171]) qui la suivent. Au cours de l'acte 1 , scène 3 , Shakespeare met en place une dynamique érotique de la querelle. L'échange entre les deux amants est comme un flot régulièrement interrompu par les protestations et injonctions d'Antoine qui tente de faire barrage aux propos de la reine. C'est dans cette résistance d'Antoine que réside le plaisir de Cléopâtre; elle constitue le moteur de la querelle. Aussi lorsque le général se dérobe en prononçant un "I'll leave you lady" (1.3.86) définitif, il détruit par là même le plaisir de la querelle. Sans lutte, point de plaisir. Cléopâtre se 
voit alors forcée de renoncer aux ré-jouissances de la dispute. Si c'est dans la résistance d'Antoine que réside le plaisir de Cléopâtre, c'est dans l'abandon de Cléopâtre qu'Antoine se trouve comblé. La querelle se révèle être ici un art érotique qui donne aux partenaires le plaisir de la victoire. On voit se profiler ici le chevauchement bien connu de l'amour et de la guerre. Énobarbus souligne cette correspondance du désir et de la querelle lorsqu'il commente le mariage d'Antoine en ces termes: "But you shall find the band that seems to tie their friendship together will be the very strangler of their amity: Octavia is of holy, cold, and still conversation" (2.6.118-20).

Pas d'union sans querelle. Dans cette pièce, le reproche est d'or ("good rebuke" [3.7.25]). La proximité de l'accord et du désaccord est suggérée dramatiquement par l'alternance de scènes de haine et de scènes d'amour entre les deux héros éponymes. "I am quickly ill, and well, / So Antony loves" (1.3.72-73): dans un monde où les personnages changent d'humeur en un clin d'œil, il n'est pas étonnant que querelles et réconciliations se succèdent à un rythme effréné. Ces retournements de la concorde en discorde sont suggérés sur un mode plus symbolique lorsque Cléopâtre commente l'anecdote de la pêche narrée par Charmian. Il faut noter que Shakespeare transforme ici la source ${ }^{36}$ pour faire de cet épisode un pari ("wager" [2.5.16]), le jeu amoureux reposant dès lors sur une forme de compétition, un mélange de conflit et de complicité. L'évocation de cet incident réveille chez Cléopâtre le souvenir des moqueries infligées à Antoine:

That time? - O times! -

I laughed him out of patience; and that night

I laughed him into patience, and next morn,

Ere the ninth hour, I drunk him to his bed;

Then put my tires and mantles on him, whilst

I wore his sword Philippan. (2.5.18-23)

"I laughed him out of patience ... I laughed him into patience": le dramaturge condense en quelques mots le schéma de flux et de reflux qui structure la pièce. Le spectateur peut relever ici deux métamorphoses: il voit le déplaisir se changer en plaisir en des termes qui suggèrent un érotique va-et-vient ("out of," "into"). Ce glissement souligne la proximité de la discorde et de l'harmonie et contient toute l'alternance structurelle qui caractérise cette pièce. On assiste également à la métamorphose du féminin en masculin. Antoine est ici dépossédé de son attribut viril et l'on peut lire dans les tortures verbales et physiques qu'il

36. Voir note de Bevington, 131. 
inflige à Thidias une tentative de resexualisation. La querelle et la provocation verbale font partie d'un jeu de rôles érotique à multiples facettes. Être l'un contre l'autre, c'est aussi être l'un tout contre l'autre.

Le dramaturge fait également de la querelle opposant Antoine et César une discorde harmonieuse, notamment en utilisant l'ambivalence du terme "competitor." ${ }^{\text {37 }}$ C'est ce que note Thomas McAlindon dans Shakespeare's Tragic Cosmos:

As many editors observe, the word 'competitor' is used in the play in the now obsolete sense of "partner', 'comrade', 'equal'. What they do not observe is that the other sense is present too, so that the word functions as an oxymoronic pun pointing to the fact that friendship-and-equality is potential strife-and-domination. ${ }^{38}$

De la même manière qu'Antoine et Cléopâtre sont deux amants qui se déchirent, Antoine et César sont deux amis qui "l'un l'autre se font grand mal"39: "two friends / That does afflict each other" (3.6.80-81). Cette formule d'Octavie prolonge la déclaration d'amour ambiguë qu'Antoine fait à César pour sceller leur réconciliation: "I'll wrestle with you in my strength of love" (3.2.63). La métaphore traduit ici l'ambivalence de la relation entre les deux chefs. Le style va contre l'idée. La compétition qui se joue entre les deux généraux romains rejoint l'harmonie discordante qui caractérise le couple éponyme. Elle est au croisement du conflit et de la collaboration, de l'empoignade et de l'embrassade: "Look, here I have you [Embracing him]" (3.2.64). Cette expression d'Antoine, que l'on peut traduire par "vois, je te tiens," est également à double tranchant: elle suggère une prise de pouvoir ou même une prise de possession tout autant qu'une invitation à l'étreinte. La même ambiguïté apparaît lorsque les festivités battent leur plein sur la galère de Pompée. L'intervention de Ménas inscrit alors la discorde au cœur de la concorde. ${ }^{40} \mathrm{Ce}$ qui est suggéré par l'action dramatique transparaît au détour d'une réplique lorsque Énobarbus invite la compagnie à entonner une chanson:

All take hands.

Make battery to our ears with the loud music,

The while I'll place you; then the boy shall sing.

The holding every man shall bear as loud

As his strong sides can volley. (2.7.102-06)

37. 1.4.3, 2.7.66, 5.1.42.

38. McAlindon, Cosmos 233.

39. Traduction de Thomas.

40. C'est un peu le même procédé dramatique que dans la scène du bal dans Roméo et Juliette. 
Du chant cordial à l'agression militaire, il n'y a qu'un pas à faire, qui est suggéré ici par l'emploi des termes "battery" et "volley." Chant de liesse et chanson militaire se confondent. Dans un monde où même le vin fraternel est qualifié de "conquérant" ("conquering wine" [2.7.101]), il n'est pas surprenant que l'accord dégénère en une étrange cacophonie. Au cours de la même scène Shakespeare crée également une discordance harmonieuse en substituant à l'affrontement provisoirement évité une compétition de buveurs. "This is not yet an Alexandrian feast" (2.7.89): cette réplique de Pompée résonne comme un défi aux oreilles d'Antoine qui, en buveur grandiose, s'empresse d'y répondre ("Strike the vessels, ho!" [2.7.90]). L'aigreur de la querelle se mêle à la douceur du vin lorsque Pompée lance à Antoine "I'll try you on the shore" (2.7.120), défi dont on ne sait plus s'il s'adresse à l'adorateur de Bacchus ou au chef de guerre. Le voile de convivialité masque à peine la querelle lorsque les deux hommes quittent la scène sur ces mots:

POMPEY

O Antony,

You have my father's house. But what? We are friends.

Come down into the boat.

ENOBARBUS

Take heed you fall not. (2.7.121-23)

En rappelant à Antoine qu'il est un "coucou," Pompée ravive une querelle avant de l'évacuer, pour un temps seulement. La mise en garde d'Énobarbus souligne l'équilibre fragile qui s'est instauré. Le vin qui coule à flot au cours de cette scène incarne bien l'ambivalence que nous venons de décrire: l'ivresse est mère de querelles, ${ }^{41}$ mais elle peut aussi plonger dans un délicieux oubli ("soft and delicate Lethe" [2.7.102]). Mais si Antoine veut oublier les querelles passées, le dramaturge s'emploie quant à lui à les inscrire au cœur de cette scène de liesse.

Ce mélange aigre-doux se manifeste également tout au long de la pièce à travers l'économie paradoxale de l'éloge et de l'injure qui y est mise en place. Dans Antony and Cleopatra, compliments et insultes se chevauchent et s'annulent, faisant place à un vide qui plonge le spectateur dans un état de perplexité. Ce mélange étonnant est des plus spectaculaires lorsqu'il est véhiculé par cette querelle de mots qu'est l'oxymore. Appelée "Royal wench" (2.2.236), Cléopâtre devient une querelle vivante. Le surnom dont Antoine affuble Cléopâtre ("My serpent of old Nile" [1.5.26]) est tout aussi ambivalent, et nombre de jugements

41. C'est l'un des lieux communs qui reviennent dans les textes puritains de l'époque et qui apparaissent chez Shakespeare notamment dans IHenry IV, La Nuit des Rois, Othello ou La Tempête. 
émis par les personnages sont à mi-chemin entre l'éloge et l'injure. Les arguments de l'accusation et de la défense sont souvent paradoxalement les mêmes. Lorsque Énobarbus décrit Cléopâtre comme une merveilleuse œuvre d'art, son propos apparemment élogieux est travaillé par un contrediscours cynique:

ANTONY

Would I had never seen her!

ENOBARBUS

O sir, you had then left unseen a wonderful piece of work, which not to have been blest withal would have discredited your travel. (1.2.147-50)

Oscillant entre chef-d'œuvre artistique et attraction touristique, ${ }^{42}$ Cléopâtre apparaît déjà comme cet objet de spectacle qu'elle refuse de devenir à la fin de la pièce, à mi-chemin entre le monstre et la merveille. ${ }^{43}$ Cette concomitance est également suggérée par la double utilisation du terme "morsel." Si Cléopâtre l'associe tout d'abord à la gloire et la beauté de sa jeunesse perdue, se rappelant le temps où elle était un "morceau de choix" ("a morsel for a monarch" [1.5. 32]), Antoine quant à lui fait de ce terme une véritable injure lorsqu'il lui rappelle ses débauches passées : "I found you as a morsel cold upon / Dead Caesar's trencher; nay you were a fragment/ Of Cneius Pompey's" (3.13.119-21). De "delicatessen," Cléopâtre se trouve reléguée au statut de piètre pitance. Sa disgrâce aux yeux d'Antoine est exprimée par le revirement sémantique du terme "morsel" dans une pièce où la nourriture et le sexe sont souvent associés. Cependant cet effet d'écho ironique suggère également une union derrière cet écart, puisque le terme de l'un est recyclé par l'autre. L'économie complexe qui s'instaure entre l'éloge et l'injure transparaît également lors de l'échange suivant:

CHARMIAN

$$
\text { O, that brave Caesar! }
$$

Cleopatra

Be choked with such another emphasis!

Say, 'the brave Antony'.

CHARMIAN

The valiant Caesar!

Cleopatra

42. Voir Linda Charnes, Notorious Identity, Materializing the Subject in Shakespeare (Cambridge, MA: Harvard UP, 1995) 121.

43. Voir Antoine qui, dans ses malédictions, la condamne à devenir un monstre de foire: "most monster-like be shown" (4.12.36). 


\author{
By Isis, ${ }^{44}$ I will give thee bloody teeth \\ If thou with Caesar paragon again \\ My man of men. \\ ChARMIAN
}

I sing but after you.

By your most gracious pardon, Cleopatra

My salad days,

When I was green in judgement, cold in blood,

To say as I said then. (1.5.70-78)

L'éloge est ici injurieux et devient source de querelle. En louant César, Charmian insulte Antoine et, par ricochet, offense sa maîtresse Cléopâtre. Porter l'un aux nues revient à rabaisser l'autre. Cléopâtre évoque le même schéma de balancier lorsqu'elle déclare: "In praising Antony, I have dispraised Caesar" (2.5.109). ${ }^{45}$ Dans un monde où règne l'hyperbole, la louange ne se partage pas et la comparaison n'est pas de mise. C'est le principe d'exclusivité qui prévaut. Superlatifs et comparatifs sont incompatibles. La réaction violente de Cléopâtre fait oublier un instant que c'est de César et non d'Octave qu'il s'agit ici. Ainsi, derrière cette concurrence de Jules César et d'Antoine on aperçoit les reflets de la compétition entre Octave et Antoine. Au détour d'une querelle badine entre les deux femmes, on entrevoit l'idée, si finement soulignée par Ian Kott, ${ }^{46}$ que le monde est trop petit pour ces deux chefs. Derrière la compétition amoureuse se cache la concurrence politique. Il n'y a pas de place pour deux dans le cœur de Cléopâtre; il n'y a pas de place pour deux dans le cœur des Romains. Shakespeare inscrit à nouveau l'injure au cœur de l'éloge lorsque Antoine se plaint de ce que les louanges de César aient été par trop mesurées:

[He] spoke scantly of me.

When perforce he could not

But pay me terms of honour, cold and sickly

He vented them, most narrow measure lent me;

When the best hint was given him, he not took't,

Or did it from his teeth. (3.4.5-10)

Shakespeare met ici en tension la manière et la matière du discours. Si la matière est élogieuse ("terms of honour"), la manière est quant à elle

\footnotetext{
44. Au sujet du mythe d'Isis et d'Osiris qui selon McAlindon cst une version de la "discordia concors," voir Cosmos 230.

45. Pour ce schéma, voir aussi "You praise yourself / By laying defects of judgement to me" (2.2.60-61) et "Thy lustre thickens / When he shines by" (2.3.27-28).

46. Ian Kott, Shakespeare our Contemporary (London: Methuen, 1965) 134-40.
} 
injurieuse, tant elle pèche par manque d'excès. Lorsque l'éloge n'est pas à la mesure de son sujet, il en devient insultant. ${ }^{47}$

Le dramaturge suggère également la conjonction de la concorde et de la discorde en faisant glisser le discours de la critique à la louange ou, inversement, de l'apologie à la diatribe. Lorsque Antoine enjoint à sa reine de cesser de lui chercher querelle au cours d'une réplique citée plus haut, son discours se métamorphose presque imperceptiblement et suit un parcours qui nous mène de "fie" (1.1.50) à "fair" (1.1.53). Ce saut d'un terme à l'autre est révélateur des paradoxes propres à la querelle. Dans une pièce où le défaut est perfection, il n'est pas étonnant que la querelle soit harmonie. L'éloge funèbre d'Octave à Antoine, le frère ennemi, est travaillé par ce paradoxe. Tout au long de la pièce Octave raconte Antoine tel qu'il était et tel qu'il est devenu. Admirateur d'un Antoine passé, il est aussi le détracteur de l'Antoine nouveau. Or ces deux discours se rejoignent dans cet éloge funèbre au cours duquel Octave tente désespérément d'écrire sa propre légende. Si au cours de la pièce l'histoire d'Antoine et de Cléopâtre est écrite et réécrite par les autres personnages ("the world's report" [2.3.5]), César est le seul quant à lui à écrire la sienne. Les portraits sommaires que Cléopâtre et le devin dressent de lui tendent à le ridiculiser plutôt qu'à le grandir. Pour Cléopâtre, il n'est qu'un jeunot sans envergure ("scarce-bearded Caesar" [1.1.22]), tandis que le devin fait de lui un chanceux et non le conquérant héroïque qu'il prétend être (2.3.17-30). Au cours de cet éloge funèbre, César s'emploie à s'approprier la mort de son adversaire, alors que Shakespeare fait tout pour en brouiller les motifs: "O Antony, / I have followed thee to this" (5.1.35-36), "I must perforce / Have shown to thee such a declining day" (37-38), "we could no stall together / In the whole world" (39-40). Or se présenter comme vainqueur revient à rabaisser Antoine au rang de perdant. D'autre part, cet éloge masque à peine un autoportrait, comme le suggère la récurrence du pronom "I" et des adjectifs possessifs "my" et "mine." Si ce discours semble contribuer à grandir le défunt Antoine ("so great a thing" [5.1.14], "not a single doom" [5.1.18], "a moiety of the world" [5.1.19]), il sert en fait davantage à promouvoir César. En même temps que ce vainqueur sans victoire dresse le portrait mythique d'un ami, il démembre le corps d'un ennemi: ${ }^{48}$ Antoine devient en effet une maladie (5.1.36-37) et n'est plus ensuite qu'un morceau du corps gigantesque de César (45-46). Dans une scène qui suggère une sorte de cannibalisme symbolique, Octave se nourrit du

47. On retrouve la même logique paradoxale quand le messager, pris de peur, dit à Cléopâtre: "Take no offence that I would not offend you" (2.5 .101).

48. Voir Adelman, Suffocating Mothers 184. 
corps de son ennemi, s'en approprie la force. Pour Antoine, qui se décrit comme le plus grand prince du monde ("the greatest prince o'th'world" [4.15.56]), être la moitié du monde, c'est aussi n'être que la moitié du monde. Ce n'est pas le moindre des paradoxes que l'hyperbole puisse contenir en creux une réduction. Le discours de César offre ces deux points de vue. Enfin dire que cette mort devrait faire plus grand bruit ("The breaking of so great a thing should make / A greater crack" [5.1.1415]), c'est aussi souligner qu'elle n'en fait pas. Ainsi ce discours est à double tranchant. En tentant d'écrire une histoire d'amitié, César raconte en fait l'histoire d'une querelle. Cette réconciliation posthume n'est que la réécriture des querelles passées. Omniprésente, envahissante, apparemment transparente, la querelle devient insaisissable et opaque tant elle donne lieu à de nombreuses réécritures.

\section{"What's the quarrel?" 49}

Reprenant la distinction opérée par César à propos des commentaires quelque peu sarcastiques d'Énobarbus, nous pourrions dire que dans Antony and Cleopatra, la querelle est une affaire de "manière" dont la "matière" échappe toujours car elle s'écrit et s'efface, se remplit et se vide en permanence. Le fond des querelles est un fond mouvant ("quicksands" [2.7.55]), changeant, bouillonnant. L'oraison funèbre que nous venons d'évoquer traduit le brouillage qui s'opère tout au long de la pièce entre querelle, guerre et compétition, querelle privée et conflit politique. Ce brouillage apparaît tout d'abord à travers le traitement réservé aux médiateurs dans cette pièce. Si l'envoi de messagers inscrit la querelle dans une logique diplomatique, les réactions excessives d'Antoine et de Cléopâtre aux messages envoyés tirent la querelle vers le domaine privé. Les mauvais traitements infligés aux messagers, et notamment à Thidias, traduisent une confusion entre conflit politique et querelle privée. Si César semble s'en tenir à une perspective politique, Antoine quant à lui glisse dans le règlement de compte personnel. Le défi qu'il envoie à Octave est révélateur de ce décalage:

I dare him therefore To lay his gay caparisons apart And answer me declined, sword against sword, Ourselves alone. (3.13.25-28)

49. Shakespeare, Troilus and Cressida, ed. Kenneth Muir (Oxford: Oxford UP, 1994) (2.1.87). 
Par ce défi d'homme à homme, Antoine entend vider une querelle privée; en refusant ce défi César choisit la guerre plutôt que la querelle:

He calls me boy, and chides as he had power

To beat me out of Egypt. My messenger

He hath whipped with rods, dares me to personal combat,

Caesar to Antony. Let the old ruffian know

I have many other ways to die, meantime

Laugh at his challenge. (4.1.1-6)

La querelle s'inscrit entre les deux pôles que sont le duel et la guerre.

Shakespeare brouille également les contours de la querelle en suggérant à plusieurs reprises le télescopage du mot et de l'acte. C'est tout d'abord Antoine qui confond le dire et le faire/fer lorsqu'il s'exclame "Would we had spoke together!" (2.2.176). Même si ce cri du cœur est ambigu, l'une des interprétations possibles est "would we had exchanged blows, joined battle," ${ }^{10}$ la parole rejoignant alors l'acte. La même ambivalence ressort de l'échange entre César et Pompée un peu plus tard:

\section{POMPEY \\ Your hostages I have, so have you mine, And we shall talk before we fight. \\ CAESAR}

Most meet

That first we come to words... (2.6.1-3)

Si Pompée dissocie le mot et l'acte en mettant en relief un ordre chronologique, l'expression "come to words" utilisée par César contient en creux l'expression plus courante "come to blows" et trahit ainsi la superposition de la parole et de l'action. Cet effet de brouillage apparaît de façon encore plus explicite quand Antoine renchérit en disant "We'll speak with thee at sea" (2.6.25), signalant ainsi son désir d'en découdre avec Pompée non seulement en paroles mais en actes. De la querelle à la guerre, il n'y a qu'un minuscule pas à faire. Les mots sont des actes en puissance. Les mots contiennent les actes.

Si la querelle se dissout dans l'acte, elle se dissout également dans la prolifération. À plusieurs reprises les personnages signalent qu'il est un temps pour la querelle. C'est tout d'abord le cas de Lépide lorsqu'il souligne qu'il est d'autres affaires à régler que ces querelles intestines et qu'il faut passer outre aux susceptibilités personnelles: "'Tis not a time for private stomaching" (2.2.9). Un peu plus tard Pompée note que si César

50. Voir note de Bevington, 122. 
et Antoine n'avaient pas d'ennemis extérieurs, ils se déchireraient entre eux:
Were't not that we stand up against them all,
'Twere pregnant they should square between themselves,
For they have entertainèd cause enough
To draw their swords. But how the fear of us
May cement their divisions and bind up
The petty difference, we yet not know. (2.1.45-50)

Les enjeux nationaux importants ("greater matters" [2.2.11]) priment (ou devraient primer) sur les querelles futiles appelées tour à tour "lesser enmities" (2.1.44), "petty difference" (2.1.50), "petty war" (2.1.34), "trivial difference" (2.2.23), "little jealousies" (2.2.140) et qui sont décrites comme le fruit de l'oisiveté ("You shall have time to wrangle in when you have nothing else to do" [2.2.112-13]). On voit se dessiner un ordre de priorité. On pourrait dire que la pièce bascule lorsque l'ennemi extérieur est écarté et que les chefs romains peuvent se livrer en toute quiétude à leur jeu favori: la querelle ou la compétition. Cependant, si les questions nationales doivent dépasser les disputes vaines, dramatiquement ce sont ces querelles qui envahissent l'imaginaire de la pièce. Shakespeare suggère sans doute qu'il est un temps pour la querelle mais il s'emploie aussi à souligner qu'elle est de tous les temps. Les querelles que, comme Lépide, on essaie d'étouffer n'en sont pas pour autant effacées mais elles évoluent au contraire sur le mode du palimpseste en donnant lieu à un perpétuel processus de réécriture. Le motif de la querelle disparaît alors derrière une multitude de motifs. La scène du conseil du triumvirat nous montre un monde où l'on "cherche" querelle au sens propre, où l'on court après ce "word of war" tout en essayant de l'éviter. Au cours de cette scène, la querelle se raye et se réécrit en permanence. La matière de la querelle y est travaillée de multiples façons. Elle est tout d'abord écrite, imaginée, scénarisée par Lépide et Énobarbus qui nous en donnent deux versions opposées (2.2.114). Puis les motifs de querelle se succèdent et sont raturés, rayés l'un après l'autre par les intervenants: "If you'll patch a quarrel, /As matter whole you have to make it with, / It must not be with this" (2.2.58-60), "Let this fellow / Be nothing of our strife" (2.2.85-86), "if we contend, / Out of our question wipe him" (2.2.86-87). La querelle se construit par couches successives. Plus on essaie de l'effacer, plus elle apparaît et l'on trouve toujours dans l'une les traces d'une autre. Pour reprendre le vocabulaire judiciaire, les chefs d'accusation sont si nombreux et si changeants que la querelle devient protéiforme, hybride, insondable. Cette dissémination des motifs de querelle apparaît au cœur de l'acte 3. 
"Nay, nay, Octavia, not only that" (3.4.1): cette réplique qui ouvre la scène 4 de l'acte 3 inscrit la querelle dans une logique de surenchère. Les motifs de querelle apparaissent d'emblée infinis ("not only"). Il semble qu'un motif en cachera toujours un autre. Antoine avance des griefs dont on ne sait vraiment s'ils sont d'ordre politique ou personnel. Il critique en effet une initiative diplomatique ("he hath waged/ New wars 'gainst Pompey" [3.4.3-4]) tout en dénonçant l'atteinte que César fait subir à son honneur ("If I lose mine honour, / I lose myself" [3.4.22-23]). Cette incertitude quant à la nature de la querelle se traduit par l'indétermination de la formule utilisée par Antoine: "When it appears to you where this begins" (3.4.33). Le spectateur ne peut, pas plus qu'Octavie, donner forme à ce "this" dont les contours sont redéfinis à l'envi. Cet effet de dissémination est prolongé à l'acte 3 , scène 6 , lorsque les deux parties énumèrent leurs griefs respectifs. "Contemning Rome, he has done all this and more" (3.6.1): cette formulation suffit à évoquer à nouveau une longue liste de griefs. Ceux que César énumère au cours de la scène (3.6.1-19) ne peuvent apparaître dès lors que comme le sommet de l'iceberg. Les motifs d'Antoine tels qu'ils sont rapportés par César contredisent en partie ceux que le général avançait deux scènes auparavant. La querelle change encore de profil lorsque César voit sa sœur délaissée pour un monde de plaisirs: "You are abused / Beyond the mark of thought" (3.6.89-90). Généreux en toute chose, Antoine ne peut que générer une multitude d'offenses ("most large / In his abominations" [3.6.97-98]). Par ailleurs, la querelle est d'autant plus proliférante qu'elle est médiatisée et commentée dans cette pièce. À l'acte 3 , scène 5, Éros réécrit la querelle qui vient de nous être présentée par Antoine. Le dramaturge gonfle le poids dramatique du défi lancé par Antoine en nous présentant son émission (3.13.25-28) mais aussi sa réception (4.1.1-6), ainsi que les commentaires qu'il suscite $(3.7 .30-34 ; 3.13 .29-37 ; 4.4 .36-7)$.

La querelle se développe par strates successives et l'on perçoit à tout moment les traces de rivalités et de disputes anciennes. En reprochant à Antoine d'avoir spolié son père $(2.6 .27-29 ; 2.7 .122-23)$, Pompée inscrit une querelle nouvelle dans une lignée. Le présent porte le poids d'un héritage conflictuel. La querelle relie et relit hier et aujourd'hui. Lorsque Pompée, au cours de la même scène, rappelle à Antoine que sa reine est passée par d'autres mains et notamment par celles de Jules César (2.6.6265), il relègue la querelle à un temps révolu, tandis que la réaction d'Antoine ancre ce passé dans le présent. L'allusion de Pompée trouve en effet un puissant écho lorsque Antoine, furieux, rappelle à Cléopâtre ses 
débauches d'autrefois ("Triple-turned whore" [4.12.13]). ${ }^{51}$ Enfin, l'évocation de Césarion $(3.6 .6 ; 3.13 .166)$, fils présumé de Jules César et de Cléopâtre, résume les tensions léguées par les anciens. Les contours de la querelle se font également indistincts lorsque Shakespeare en mêle les fils. La figure de César (Jules et/ou Octave) hante les querelles entre Antoine et Cléopâtre et la figure de Cléopâtre hante les querelles entre Antoine et Octave. Ces effets de croisement dessinent une véritable compétition entre Octave et Cléopâtre, Antoine devant choisir entre deux types d'unions finalement toutes deux mues par le conflit. Qu'il s'agisse des rapports entre Octave et Antoine ou entre les deux amants, la querelle est en effet affaire de désir. "[P]eace or war, / As thou affects" (1.3.7071): lorsque Antoine laisse à Cléopâtre le choix de la paix ou de la guerre, il souligne que la querelle repose sur le désir. Cet aspect irrépressible est exprimé d'une autre façon par Énobarbus lorsqu'il décrit les rapports des deux chefs romains en ces termes: "Then, world, thou hast a pair of chaps, no more; / And throw between them all the food thou hast, / They'll grind the one the other" (3.5.11-13).

Cette image qui évoque deux êtres irrémédiablement liés l'un à l'autre mais inévitablement voués à se dévorer convient tout autant au couple Octave-Antoine qu'au couple Antoine-Cléopâtre.

"When it appears to you where this begins" (3.4.33): en quittant Octavie, Antoine lui livre une énigme impossible à résoudre. Impossible en effet de savoir où "cela" commence, tant les contours du conflit sont polymorphes, brouillés, insaisissables. Il pourrait sembler plus facile de découvrir où "cela" finit ("where this ends"), la mort d'Antoine et de Cléopâtre paraissant mettre un terme à une querelle en ouvrant sur une ère de paix universelle ("universal peace" [4.6.5]). Cependant, l'oraison funèbre d'Octave, par son ambivalence, raconte une histoire d'amitié autant que de querelle, et la mort de Cléopâtre semble la prolonger au moins à deux titres. Cette mort, qui est en soi un affront fait à César, est d'autant plus provocatrice aux yeux de spectateurs élisabéthains pour qui l'expression "Fig!" était hautement injurieuse. L'utilisation des figues résonne alors comme une littéralisation ou une réification de l'injure faite à César, un dernier pied de "nez" au futur Auguste. D'autre part le "ass unpolicied" (5.2.301-302) que Cléopâtre fait dire à son serpent est à l'image des querelles fantômes qui habitent cette pièce de leur absenceprésence; elle est comme une injure venue d'outre-tombe qui viendra hanter les plus beaux jours d'Auguste.

51. Voir aussi 3.13.119-23. 
Antony and Cleopatra est bâtie sur une querelle paradoxale qui est à la fois vidée et saturée de contenu, stérile et féconde, constamment éliminée et alimentée, digérée ("digested" [2.2.188]) et nourrie en permanence. À l'issue de ce parcours, on cerne mieux les implications des deux expressions citées au début de notre étude. Dire d'Antoine qu'il est "the word of war" ou "the merèd question," c'est transformer un personnage en matière à querelle, une matière qui est sculptée, tout au long de la pièce et qui, comme les nuages évoqués par Antoine (4.14.114), se métamorphose sans jamais disparaître, se dissout pour mieux se reconstruire, oscille entre le réel et l'imaginaire. "All's but naught" (4.15.83): cet aphorisme lancé par Cléopâtre résonne comme une définition de cette "matière" qui, lorsque l'on parvient à en faire un tout ("As matter whole you have to make it with" [2.2.59]) se décompose pour n'être plus que "rien."

Nathalie VIENNE-GUERRIN

Université de Rouen 